\title{
EFECTO DEL ÁCIDO INDOL 3 BUTÍRICO Y CASCARILLA DE ARROZ CARBONIZADA EN EL ENRAIZAMIENTO DE ESTAQUILLAS DE CAOBA (Sw ietenia macrophylla) EN CÁM ARAS DE SUB-IRRIGACIÓN EN LA AMAZONÍA PERUANA
}

\author{
Federico YEPES ${ }^{1}$, Dennis DEL-CASTILLO ${ }^{1}$, Manuel SOUDRE ${ }^{1}$, Julio SOPLIN ${ }^{1}$, Jack CHUNG ${ }^{1}$ \\ 1 Instituto de Investigaciones de la Amazonía Peruana, Programa Probosque, Av. Abelardo Quiñónez km 2.5, Iquitos, Perú, E-mail: \\ fyepes@ iiap.org.pe
}

\section{RESUMEN}

El propósito del estudio fue determinar el efecto de diferentes concentraciones de ácido indol 3 butírico (AIB) y sustratos (arena y cascarilla de arroz carbonizada) en el enraizamiento de estaquillas juveniles de la Swietenia macrophylla (caoba). Las estaquillas procedieron de brotes apicales juveniles y con dimensiones de $6 \mathrm{~cm}$ de longitud y $40 \mathrm{~cm}^{2}$ de área foliar. Se procedió a realizar el estudio en dos ensayos consecutivos. En el primero se probaron diferentes sustratos y dosis de ácido indol butírico, y en el segundo, se probaron dos tipos de estaquillas (apical y basal) con el mejor resultado de la interacción de sustrato y dosis del primer ensayo. Se utilizó la cámara de sub-irrigación como microambiente de propagación. El análisis de varianza $(\mathrm{p}<0.05)$ para enraizamiento y supervivencia indican que no existen diferencias significativas entre sustratos, ni en las dosis de AIB. En los sustratos arena y cascarilla carbonizada de arroz se obtuvieron $85 \%$ y $83 \%$ de enraizamiento, respectivamente. Con las dosis $0,0.1,0.3,0.5$ y $0.7 \%$ se alcanzaron $75 \%, 90 \%, 87 \%, 78 \%$ y $92 \%$ de enraizamiento respectivamente. Sin embargo, la interacción conformada por cascarilla de arroz carbonizada, $0.7 \%$ de AIB y estaquillas de tipo apical, aparentemente muestran mejores resultados. En la cuarta semana, se obtuvo un enraizamiento de $50 \%$ y en la octava de $93 \%$.

PALABRAS CLAVE: Propagación vegetativa, estaquillas, enraizamiento, AIB, sustratos, caoba, Swietenia macrophylla.

\section{EFFECTS OF INDOLE 3 BUTYRIC ACID AND BURNT RICE HUSKS ON THE ROOTING OF YOUNG SPROUTS OF MAHOGANY (Sw ietenia macrophylla) IN SUB-IRRIGATED CHAMBER IN THE PERUVIAN AMAZON}

\begin{abstract}
The aim of this study was to determine the effects of different concentrations of indole 3 butyric acid (AIB) and substrates (sand and burnt rice husks) on the rooting of young sprouts of mahogany (Swietenia macrophylla). The pegs were obtained from juvenile apical sprouts with $6 \mathrm{~cm}$ and $40 \mathrm{~cm} 2$ of length and leaf area, respectively. Two consecutive trials were carried out. In the first, different substrates and doses of indole 3 butyric acid (IBA) were tested, and in the second, the best substrate and dosage of the first trial were used to test two types of cuttings (apical and basal). Sub-irrigated chamber was used as propagation micro environment. The analysis of variance for rooting and survival showed no significant difference $(\mathrm{p}<0.05)$, between substrates or among dosage levels of IBA. For sand and burnt rice husks, rooting was $85 \%$ and $83 \%$ respectively. Dosages of $0,0.1,0.3,0.5$, and $0.7 \%$ IBA, yielded a rooting of $75 \%, 90 \%, 87 \%, 78 \%$ and $92 \%$ respectively. However, the most successful result was obtained with the interaction between burnt rice husks, $0.7 \%$ IBA and apical sprouts. In the fourth week, rooting was 50\% and by the eighth week, $93 \%$ of cuttings had rooted.
\end{abstract}

KEYW ORDS: Vegetative propagation, sprouts, rooting, IBA, substrates, mahogany, Swietenia macrophylla. 


\section{INTRODUCCIÓN}

La caoba (Swietenia macrophylla King) es la especie forestal maderable más importante en la amazonía peruana, por la calidad de su madera, alto precio y demanda en los mercados. Tiene una extensa distribución natural en el Perú, el rango comprende las regiones de Loreto, Amazonas, San Martín, Ucayali, Huanuco, Junín, Cusco, Madre de Dios y Puno (Ríos et al., 2002). En las últimas décadas, las poblaciones naturales sufrieron una intensa sobreexplotación debido a la extracción ilegal y no tecnificada de su madera, así como por la destrucción progresiva de su hábitat natural a causa de la conversión incontrolada de bosques en tierras de cultivo, poniendo en amenaza la supervivencia de la especie en los bosques de la Amazonía peruana. Por ello, a partir de 2003, la especie fue incluida en el Apéndice 2 de la Convención para el Comercio Internacional de Especies Amenazadas (CITES).

Una herramienta valiosa de reproducción para establecer estrategias de conservación, manejo y uso adecuado de los recursos genéticos amenazados y en peligro de extinción de especies como la caoba, es la propagación vegetativa. Es una técnica de reproducción asexual, la cual consiste en la producción de nuevos individuos a partir de una única planta donante, utilizándose diferentes partes de la planta como ramas, yemas, estacas, hojas, células, raíces u otros (Hartmann et al., 1997). Se obtiene semilla vegetativa con mayor ganancia genética en productividad en el menor tiempo posible, con lo que posibilita la replica de los mejores individuos con combinaciones genéticas únicas, lo cual no es posible mediante el uso de semillas botánicas.

La técnica es viable con el uso de estaquillas provenientes de tallos ortotrópicos juveniles de plántulas, rebrotes de tocones o setos manejados, sanos y vigorosos. Si se utiliza material fisiológicamente adulto, la técnica puede no ser exitosa (Mesén et al., 1992). El uso de los propagadores de sub-irrigación para el enraizamiento de estaquillas ha sido probado con éxito en más de cien especies de diferentes ecosistemas (Leakey et al., 1982a), se pueden obtener enraizamientos entre 70 $100 \%$ utilizando el sustrato y la concentración de AIB adecuadas. Se ha probado que el ácido indol 3 butírico (AIB) es la mejor auxina, ya que no es tóxico en un rango amplio de concentraciones y es efectivo en promover el enraizamiento en un gran número de especies, también es mucho más fotoestable que el ácido indol acético (AIA) y al ser insoluble en el agua permanece más tiempo en el sitio de aplicación manteniendo así su efectividad por periodos más largos de tiempo (Hartman \& Kester, 1976).
Con el fin de contribuir con técnicas para la conservación genética y contar con una nueva herramienta para la reforestación de la caoba en la Amazonía peruana, se desarrolló el presente estudio que tuvo por objetivo determinar el efecto de diferentes dosis de ácido indol 3 butírico (AIB) y sustratos en el enraizamiento de estaquillas juveniles utilizando como ambiente de micropropagación a la cámara de sub-irrigación.

\section{ATERIAL Y MÉTODOS}

\section{ÁREA DE ESTUDIO}

Los ensayos fueron realizados en el vivero del Centro de Investigaciones Jenaro Herrera (CIJH), del Instituto de Investigaciones de la Amazonía Peruana (IIAP). El CIJH se encuentra ubicado a $2.5 \mathrm{~km}$ al este del poblado de Jenaro Herrera ( $4^{\circ} 55^{\prime}$ S, $73^{\circ} 44^{\prime}$ E), en el distrito de Jenaro Herrera, provincia de Requena, Loreto. La temperatura anual es de $26^{\circ} \mathrm{C}$, con variaciones estacionales entre $25.1^{\circ} \mathrm{C}$ (julio) y $26.5^{\circ} \mathrm{C}$ (diciembre). La precipitación anual fue de $2724 \pm 171$ $\mathrm{mm}$. Existen dos estaciones, la seca de junio a setiembre con precipitación de menos de $180 \mathrm{~mm}$ por mes y la lluviosa entre diciembre y marzo. La humedad relativa promedio fue de $85.9 \%$, con un valor menor $(<85.5 \%)$ entre los meses de julio a octubre y un valor mayor (el 87.2\%) entre los meses de febrero y abril (SENHAMI, 2007).

\section{ÁREA EXPERIM ENTALY SISTEM A DE PROPAGACIÓN}

El área de enraizamiento estuvo cubierta por una malla Raschell (80\%) de color negro, a fin de disminuir la intensidad de luz y temperatura dentro del ambiente. Las condiciones ambientales externas medidas fueron: temperatura del aire $29^{\circ} \mathrm{C}$ y humedad relativa $85 \%$. Se utilizó como ambiente de micropropagación una cámara de sub-irrigación deseñada por Leakey et al. (1990) con algunas modificaciones en la parte interna. La estructura fue fabricada en base a madera y forrada completamente con plástico transparente tipo mica \#10. Las condiciones ambientales promedio dentro de la cámara fueron: temperatura $31{ }^{\circ} \mathrm{C}$, temperatura del sustrato $29^{\circ} \mathrm{C}$ y humedad relativa $88 \%$.

\section{MATERIAL VEGETATIVO}

Las plantas jóvenes de caoba fueron producidas de semilla botánica. Se realizó un corte en el ápice de la planta a $30 \mathrm{~cm}$ de altura para inducir la emisión de brotes laterales. A los 35 días se obtuvieron brotes de $15 \mathrm{~cm}$. Los brotes fueron dimensionados en estaquillas de $6 \mathrm{~cm}$ de longitud y $0.4 \mathrm{~cm}$ de diámetro promedio, 
con dos hojas de $20 \mathrm{~cm}^{2}$ área foliar cada una. La desinfección del material vegetativo se realizó con el fungicida Cupravit OB 21 (30 gr) diluido en 10 litros de agua. El material vegetativo fue sumergido en el desinfectante durante diez minutos y luego secado al aire libre.

\section{SUSTRATOS}

Los sustratos utilizados fueron arena de grano media (diámetro $0.2-1.0 \mathrm{~mm}$ ) y cascarilla de arroz carbonizada. El proceso de esterilización para arena consistió en el tamizado, lavado y tratada a presión de vapor de agua por dos horas. La cascarilla de arroz fue carbonizada por combustión durante cinco horas.

El proceso de carbonizado se inició con el esparcido de las cascarillas alrededor del horno metálico hasta cubrir completamente la base de acoplamiento con la chimenea formando un cono, manteniéndose una combustión constante hasta que la capa superior muestre un color oscuro como el carbón. En el proceso de carbonización se tuvo particular cuidado de evitar la formación de ceniza.

\section{PROCEDIM IENTO}

Se realizaron dos ensayos consecutivos: a) en el primer ensayo se probaron diferentes dosis de ácido indol 3 butírico (AIB) y sustratos. El AIB puro en polvo fue diluido con alcohol metílico de $96^{\circ}$ en concentraciones de $0.1,0.3,0.5$ y $0.7 \%$.

Los sustratos utilizados fueron arena y cascarilla de arroz carbonizada. b) en el segundo ensayo, se probaron dos tipos de estaquilla según la posición en el brote, el tipo apical ubicado en la parte del ápice y basal en el inicio del brote. Se utilizo el sustrato de cascarilla de arroz carbonizada, y dosis de AIB $(0.7 \%)$ obtenidos en el primer ensayo.

En ambos ensayos, las aplicaciones de AIB se realizaron remojando la base de la estaquilla por cinco segundos, de acuerdo con las recomendaciones de Hartmann \& Kester (1972) y luego ventiladas para la evaporación del alcohol. En los tratamientos se incluyó un testigo sin hormona.

Las estaquillas tratadas fueron introducidas en los sustratos dentro de la cámara de sub-irrigación, según el croquis del diseño experimental. Durante el periodo de duración del ensayo se aplicaron riegos manuales de agua por aspersión intensos en días con alta luminosidad y ligeros en los sombríos.

\section{ANÁLISIS EXPERIM ENTAL}

En ambos ensayos se utilizó un diseño de bloques completo al azar, con arreglo factorial, con diez tratamientos, tres repeticiones por tratamiento y diez unidades experimentales por repetición. Se evaluó la supervivencia de la estaquilla y la presencia de raíces. Asimismo, se registraron las variables ambientales dentro y fuera de la cámara de enraizamiento: temperatura y humedad relativa en diferentes horarios (08:00, 12:00 y 15:00). Los datos de porcentaje fueron transformados mediante la formula arcoseno $\sqrt{ } \mathrm{x} / 100$ (Snedecor \& Cochran 1980) y se sometieron al análisis con respaldo del programa estadístico SPSS. Se realizó el análisis de varianza, seguido por la prueba de Tukey $(\mathrm{p}<0.05)$ en las fuentes de variación con significación para determinar las naturalezas de las diferencias entre tratamiento.

\section{RESULTADOS}

\section{EFECTO ENTRE DOSIS DE ÁCIDO INDOL 3 BUTÍRICO (AIB) YSUSTRATOS}

En el primer ensayo, el análisis de varianza para enraizamiento y supervivencia de estaquillas, indican que no existen diferencias significativas entre los dos sustratos. El enraizamiento de caoba en arena (85\%) fue ligeramente superior que en cascarilla de arroz (83\%), aunque las pruebas de rango múltiple (Tukey) no mostraron diferencias significativas (tabla 1). De igual forma, no hubo diferencias significativas entre las dosis de AIB. Todas las dosis utilizadas favorecen la formación de callos, brotación, enraizamiento y supervivencia de estaquillas. Aparentemente, el enraizamiento de caoba en la dosis $0.7 \%$ de AIB fue ligeramente superior a las otras, aunque las pruebas de rango multiple (Tukey) no mostraron diferencias significativas. En la figura 1 se presenta el efecto de las dosis de AIB sobre el enraizamiento de caoba.

\section{EFECTO DE TIPO DEESTAQUILLAS}

En el segundo ensayo, se utilizó la cascarilla de arroz carbonizada como sustrato y la dosis de $0.7 \%$ de AIB por ser la interacción con el mejor resultado en el primer ensayo. El análisis de varianza determino diferencias significativas $(\mathrm{P}<0.05)$ en el enraizamiento por tipo de estaquillas. Las pruebas de rango múltiple (Tukey) indican que existen diferencias significativas, siendo el tipo de estaquilla apical la que obtuvo el mayor enraizamiento con relación a la estaquilla de tipo basal (Tabla 2). 
Tabla 1. Prueba de Tukey para sustratos sobre la formación de raíces en estaquillas de caoba en J enaro Herrera, Loreto.

\begin{tabular}{|l|c|c|}
\hline \multicolumn{1}{|c|}{ SUSTRATO } & ENRAIZAMIENTO(\%) & SOBREVIVENCIA(\%) \\
\hline Arena & $85 a$ & 93 \\
Cascarilla arroz carbonizada & $83 a$ & 97 \\
\hline
\end{tabular}

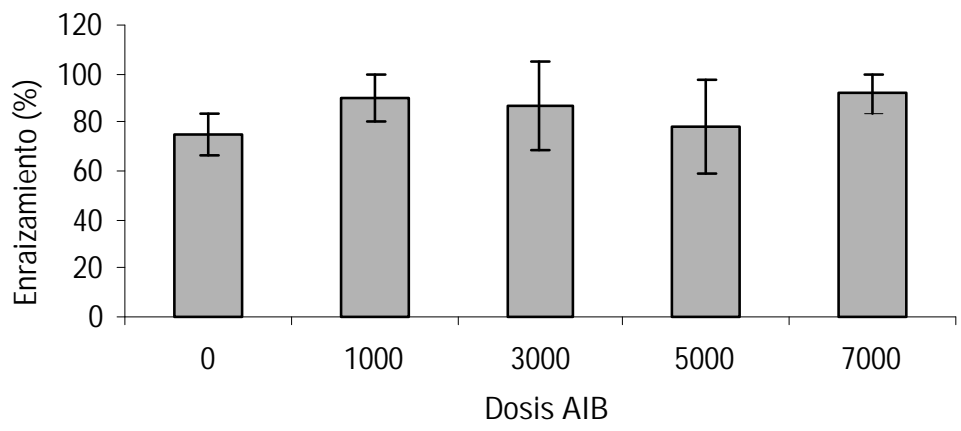

Figura 1. Efecto de la concentración de AIB sobre el enraizamiento de estaquillas de caoba Swietenia macrophylla, en J enaro Herrera, departamento de Loreto.

Tabla 2. Prueba de Tukey según tipo de estaquillas en la formación de raíces en caoba Swietenia macrophylla en J enaro Herrera, Loreto.

\begin{tabular}{|c|c|c|}
\hline TIPO ESTAQUILLA & ENRAIZAMIENTO (\%) & SIGNIFICACIÓN \\
\hline Apical & 90 & $\mathrm{a}$ \\
Basal & 55 & $\mathrm{~b}$ \\
\hline
\end{tabular}

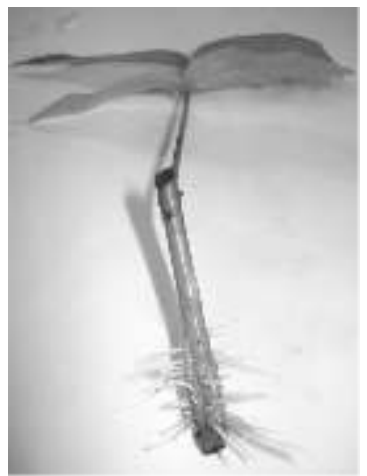

Figura 2. Fotografía mostrando el enraizamiento de caoba Swietenia macrophylla (estaquilla de tipo apical con dosis de $0.7 \%$ de AIB), obtenido en el vivero del Centro de Investigaciones J enaro Herrera, Loreto. 


\section{DISCUSIÓN Y CONCLUSIÓN}

Los valores obtenidos en ambos sustratos fueron estadísticamente similares, los resultados en arena aparentemente fueron ligeramente superiores a la cascarilla carbonizada. Sin embargo la velocidad de enraizamiento fue mayor en cascarilla carbonizada, presentándose en la cuarta semana hasta un $50 \%$ de enraizamiento. El uso de cascarilla de arroz carbonizada como sustrato permite mejores condiciones de aireación, drenaje y proporciona el soporte necesario para fijar las estaquillas y no necesita de tratamientos químicos adicionales para la esterilización; pues esta fue esterilizada en el proceso de carbonización. Los resultados reafirman lo expuesto por Díaz et al. (1992), quienes indican que el medio ideal para enraizamiento es aquel que permite buena aireación, se drene bien y sea fácil de esterilizar y que además, proporcione un soporte adecuado a la estaquilla.

Aparentemente los valores de enraizamiento obtenidos en el estudio fueron superiores a otros estudios realizados en Meliaceas. Mesén et al. (1992) y Dos Santos (2002) trabajando con Swietenia macrophylla ,y Díaz et al. (1992) trabajando con Cedrela Odorata reportan que en diferentes concentraciones de AIB $(0,0.2,0.4,0.8$ y $1.6 \%)$ disuelto en metanol obtuvieron valores de enraizamiento que varían entre el $40 \%$ y $76 \%$ utilizando como sustratos grava, mezcla de grava y aserrín, arena y mezcla de arena aserrín.

El alto valor de enraizamiento $(90 \%)$ obtenido en las estaquillas de tipo apical, ratifica la presencia de altas concentraciones de auxinas en la parte meristemática. Esto es concordante con lo reportado por Zeiger \& Taiz (2007), quienes indican que si bien las auxinas se encuentran en toda la planta, las mayores concentraciones se localizan en las regiones meristemáticas apicales en crecimiento activo, trasladándose en forma basipétala desde el punto apical de la planta hacia la base. Este flujo de auxina reprime el desarrollo de brotes axilares laterales a lo largo del tallo, manteniendo de esta forma la dominancia apical. Asimismo, mencionan que las auxinas en elevadas concentraciones promueven la formación de raíces laterales y adventicias, típico en el enraizamiento de las especies nativas amazónicas.

Estos resultados permiten concluir que el enraizamiento de caoba con estaquillas juveniles en cámaras de sub-irrigación fue exitoso. Se obtuvo resultados similares con ambos sustratos, con la diferencia que el sustrato con cascarilla carbonizada presenta una mayor velocidad de enraizamiento. Por lo tanto se recomienda a la cascarilla de arroz carbonizada como sustrato, uso de AIB en dosis de $0.7 \%$, y estaquillas de tipo apical de $20 \mathrm{~cm}^{2}$ área foliar, $5 \mathrm{~cm}$ de longitud para el enraizamiento de caoba en la Amazonía peruana.

\section{AGRADECIMIENTOS}

El presente estudio fue financiado por el Fondo para la Innovación, Ciencia y Tecnología (FINCyT) a través del proyecto: "Desarrollo tecnológico apropiado para la propagación vegetativa aplicado a la producción intensiva de semilla vegetativa de especies maderables valiosas en las regiones Loreto y Ucayali (PROVEFOR)". Los autores desean agradecer a Javier Souza Padilla y Gaspar Vilchez personal técnico del Centro de Investigaciones Jenaro Herrera por su apoyo en el trabajo de campo.

\section{BIBLIOGRAFÍA CITADA}

Díaz E.; Salazar, R.; Mesén, F. 1992. Enraizamiento de estacas juveniles de Cedrela odorata L. Revista Silvoenergia. $\mathrm{N}^{\circ}$ 51. CATIE. Turrialba. Costa Rica. 4pp.

Dos Santos, G. 2002. Propagación vegetativa de mogno, cedro rosa, jequitibá rosa y angico vermelho por miniestaquia. Monografia. Departamento de Ingeniería Forestal. Universidad de Visosa. Brasil.

Hartmann, H.; Kester, D.; Davis, J.R.F. 1997. Plant propagation: principles and practices. $6^{\mathrm{a}}$ ed. New York: Englewood Clipps / Prentice Hall, EEUU. 770pp.

INSTITUTO NACIONAL DE RECURSOS NATURALES DEL PERÚ (INRENA). 2007. Plan de acción estratégico para la implementación del apéndice II de la CITES para la caoba en el Perú (PAEC-Perú). Lima, Perú. 32pp.

Leakey, R; Chapman, V.; Longman, K. 1982. Physiological studies for tropical tree improvement and conservation. Factors affecting root initiation in cuttings of Triplochiton scleroxylon K. Schum. Forest Ecology and Management. 4:53-66.

Mesén, F.; Leakey, R.; Newton, A. 1992. Hacia el desarrollo de técnicas de silvicultura clonal para el pequeño finquero. Revista el Chasqui. N² 28:6-18. CATIE. Turrialba. Costa Rica. 12pp.

Ríos, J.; Stern, M.; León, F.; Reátegui, F. 2002. Análisis del estado de conservación de la caoba (Swietenia macrophylla) en el Perú. WWF Oficina Programa Perú. Vol. 1: Texto del informe. Lima, Perú. 99pp.

SERVICIO NACIONAL DE METEOROLOGÍA E HIDROLOGÍADEL PERÚ (SENHAMI). 2007.

Zeiger, E.; Taiz, L. 2007. Fisiología vegetal. Ciencies experimentals/ Experimental Sciences. Edición 3. Universidad de Jaume. España. 1907pp. 